\title{
Amantadine Effect on Perceptions of Irritability after Traumatic Brain Injury: Results of the Amantadine Irritability Multisite Study
}

\author{
Flora M. Hammond, ${ }^{1,2}$ Mark Sherer, ${ }^{3}$ James F. Malec, ${ }^{1}$ Ross D. Zafonte, ${ }^{4}$ Marybeth Whitney, \\ Kathleen Bell,, ${ }^{5,7}$ Sureyya Dikmen, ${ }^{5}$ Jennifer Bogner, ${ }^{6}$ Jerry Mysiw, ${ }^{6}$ and Rashmi Pershad ${ }^{2}$ \\ as the Amantadine Irritability Multisite Study Group
}

\begin{abstract}
This study examines the effect of amantadine on irritability in persons in the post-acute period after traumatic brain injury (TBI). There were 168 persons $\geq 6$ months post-TBI with irritability who were enrolled in a parallel-group, randomized, double-blind, placebo-controlled trial receiving either amantadine $100 \mathrm{mg}$ twice daily or equivalent placebo for 60 days. Subjects were assessed at baseline and days 28 (primary end-point) and 60 of treatment using observer-rated and participant-rated Neuropsychiatric Inventory (NPI-I) Most Problematic item (primary outcome), NPI Most Aberrant item, and NPI-I Distress Scores, as well as physician-rated Clinical Global Impressions (CGI) scale. Observer ratings between the two groups were not statistically significantly different at day 28 or 60; however, observers rated the majority in both groups as having improved at both intervals. Participant ratings for day 60 demonstrated improvements in both groups with greater improvement in the amantadine group on NPI-I Most Problematic $(p<0.04)$ and NPI-I Distress $(p<0.04)$. These results were not significant with correction for multiple comparisons. CGI demonstrated greater improvement for amantadine than the placebo group $(p<0.04)$. Adverse event occurrence did not differ between the two groups. While observers in both groups reported large improvements, significant group differences were not found for the primary outcome (observer ratings) at either day 28 or 60 . This large placebo or nonspecific effect may have masked detection of a treatment effect. The result of this study of amantadine $100 \mathrm{mg}$ every morning and noon to reduce irritability was not positive from the observer perspective, although there are indications of improvement at day 60 from the perspective of persons with TBI and clinicians that may warrant further investigation.
\end{abstract}

Key words: agitation; aggression; amantadine; brain injuries; irritability

\section{Introduction}

I RRITABILITY IS PRESENT in 29-69\% of persons with traumatic brain injury (TBI) in the post-acute period. ${ }^{1-3}$ Irritability adversely impacts interpersonal relationships and community integration resulting in high psychosocial and economic cost for the person with brain injury, caregivers, and society. ${ }^{1,3}$ The efficacy of pharmaceutical treatment for TBI irritability is not well-established.
Systematic evidence is needed to guide the management of this chronic and pervasive problem.

Review of the literature revealed one randomized, double-blind, controlled medication trial specifically addressing TBI irritability. Hammond and associates ${ }^{4}$ studied 76 persons at least 6 months post-TBI in a single-site, randomized-controlled trial of amantadine $100 \mathrm{mg}$ twice daily versus placebo for 28 days. Significant differences favoring amantadine were found for the observer-rated

\footnotetext{
${ }^{1}$ Department of Physical Medicine and Rehabilitation, Indiana University School of Medicine, and Rehabilitation Hospital of Indiana, Indianapolis, Indiana.

${ }^{2}$ Department of Physical Medicine and Rehabilitation, Carolinas Rehabilitation, Carolinas HealthCare System, Charlotte, North Carolina.

${ }^{3}$ TIRR Memorial Hermann, Houston, Texas.

${ }_{5}^{4}$ Spaulding Rehabilitation Hospital, Harvard Medical School, Massachusetts General Hospital, and Brigham and Women's Hospital, Boston, Massachusetts.

${ }^{5}$ University of Washington, Seattle, Washington.

${ }^{6}$ Department of Physical Medicine and Rehabilitation, The Ohio State University, Columbus, Ohio.

${ }^{7}$ University of Texas Southwestern Medical School, Dallas, Texas.
}

(c) Flora M. Hammond, Mark Sherer, James F. Malec, Ross D. Zafonte, Marybeth Whitney, Kathleen Bell, Sureyya Dikmen, Jennifer Bogner, Jerry Mysiw, Rashmi Pershad 2015; Published by Mary Ann Liebert, Inc. This Open Access article is distributed under the terms of the Creative Commons Attribution Noncommercial License (http://creativecommons.org/licenses/by-nc/4.0/) which permits any noncommercial use, distribution, and reproduction in any medium, provided the original author(s) and the source are credited. 
Neuropsychiatric Inventory Irritability (NPI-I) Most Problematic. Differences in NPI-I Observer Distress were not statistically significant between groups. Perspectives of those with TBI were not assessed. The present study aimed to replicate this study and address important questions unanswerable with the smaller study.

Through a multisite, prospective, double-blind, randomized, placebo-controlled trial, this study aimed to: (1) determine if the single-site results were reproducible; (2) assess the generalizability to different regions and centers; (3) determine if the amantadine irritability effect persisted over 60 days; and (4) evaluate participant and clinician impressions of effect. We hypothesized that amantadine (100 mg every morning and noon), compared with placebo, administered to persons with irritability at least 6 months post-TBI, would reduce irritability frequency, severity, and distress as measured by NPI at day 28 and day 60 treatment intervals.

\section{Methods}

\section{Setting}

The study was conducted at seven sites: Carolinas Rehabilitation, Carolinas Health Care System in Charlotte, NC (lead site); Indiana University School of Medicine and Rehabilitation Hospital of Indiana in Indianapolis, IN; Kessler Institute of Rehabilitation in West Orange, NJ; Spaulding Rehabilitation Hospital and Harvard Medical School in Boston, MA; TIRR Memorial Hermann in Houston, TX; The Ohio State University in Columbus, OH; and University of Washington in Seattle, WA.

\section{Study oversight}

The study was approved by the Institutional Review Board at each site and registered on www.clinicaltrials.org (\#NCT00779324). Participants and observers provided informed consent. An external Data and Safety Monitoring Board provided independent oversight. The data coordinating center stored, maintained, and transferred the data to the study statistician at study closure.

\section{Participants}

Participant recruitment occurred via referrals, letters from physicians, newsletters, and local brain injury support groups. Persons were eligible if (1) 16-75 years old, (2) sustained a nonpenetrating TBI at least 6 months before enrollment, and (3) obtained a score $\geq 6$ on Observer-rated NPI-I Most Problematic. TBI was verified by record review and clinician interview with a requirement to meet at least one of the following criteria: (1) post-resuscitation Glasgow Coma Scale (GCS) $\leq 13$; (2) GCS Motor < 6 off paralytics; (3) any period of loss of consciousness attributable to TBI; (4) post-traumatic amnesia lasting $\geq 24 \mathrm{~h}$; (5) neuroimaging consistent with TBI; and/or (6) other evidence of TBI-related focal neurological findings. An observer (family member, close friend, or employer) with adequate interaction with the person with TBI to observe irritability was required.

Enrollment was further contingent on medical and neurological stability, ability to comply with the study protocol, negative pregnancy test, and creatinine clearance $>60$. Exclusion criteria were: (1) inability to interact and communicate, (2) threat of harm to self or other, (3) diagnosis of other neurologic disorder, (4) schizophrenia or psychosis, (5) seizure in the month before enrollment, (6) concomitant use of typical neuroleptic agents or monoamine oxidase inhibitors (because of potential drug interactions), (7) previous adverse reaction to amantadine, (8) treatment with amantadine during the month before enrollment, and (9) enrollment in the previous single-site amantadine irritability study.

All psychoactive medications were on stable dosing for more than 1 month before enrollment with no plans to start or change medications during the 60-day study. Active rehabilitation therapies, behavior treatments, and counseling, if present, had been started at least 1 month before enrollment, and none started during the study. We did not attempt to record and compare groups on therapy involvement because both rehabilitation and psychological therapies may vary considerably in approach and quality among providers and hence there is no accepted method to quantify the impact of these types of therapy. Rather, we relied on random assignment to control for this and other extraneous variables between groups.

\section{Procedures}

Demographic variables, medical history, and injury data were collected by interview and verified by chart review. Measures were completed by the participant, observer, and study physician. These raters remained the same throughout the study participation. The observer rating was the primary study outcome. After baseline assessment and eligibility confirmation, Participants were randomized to receive either amantadine $100 \mathrm{mg}$ every morning and noon or to receive placebo. Weekly calls were made to assess tolerance and encourage compliance. The dose was reduced or terminated either temporarily or permanently when needed for presumed intolerance. Adverse events, changes in concomitant medications, and the remaining number of pills were recorded. Medication compliance was defined a priori as taking $80 \%$ or more of the study medication.

Outcome assessment occurred at day $28 \pm 3$ days (primary endpoint) and day $60 \pm 3$ days. Day 28 assessment was chosen to allow enough time to observe changes in the person's irritability, accommodate NPI's 4-week observation interval, and allow direct comparison with the previous single-site study. Day 60 assessment was chosen to evaluate the longer-term effect.

\section{Randomization and masking}

Group assignment occurred through computer-generated block randomization. Randomization was stratified for depression (Beck Depression Inventory-II <13 vs. $\geq 13$ ), as was also done in Hammond and associates ${ }^{4}$ single-site amantadine irritability study, to ensure balance between the two treatment groups because depression can be independently associated with irritability. Group allocation was concealed. Allocation occurred through the data coordinating center web page. Study coordinators entered eligibility criteria, and if criteria were met, the data coordinating center sent the study coordinator and local pharmacist a study number that identified which study kit to dispense. Placebo and amantadine pills were identical in taste and appearance. All participants, observers, and personnel were blinded to group assignment. Compounding pharmacist and data coordinating center had access to blinding information.

\section{Measures}

NPI-I Most Problematic and Most Aberrant. The NPI is a 40 -item rating scale developed to assess 12 behavioral domains. ${ }^{5}$ Only the NPI-I domain was used for the study. NPI-I items include: bad temper, rapid mood changes, sudden anger, impatience, crankiness, argumentative. The rater selects the frequency (1-3) and severity (1-4) of the most aberrant of these behavioral aspect(s) over the preceding month. The NPI score is the product of the frequency and severity. ${ }^{5} \mathrm{We}$ used the worst item score provided by the rater as NPI-I Most Aberrant.

In addition to deriving a NPI score that represented the most aberrant, the rater selected and rated the item most problematic to them (i.e., NPI-I Most Problematic). Analyses used both the NPI Most Problematic (primary efficacy outcome) and NPI Most Aberrant (secondary post hoc analysis).

The NPI was designed to record caregiver impressions only. We developed a version of the NPI-I to also capture participant 
impressions. Because of the complexity of this task and expected difficulties of persons with TBI in memory and executive functions, we asked the raters to indicate the frequency and severity of each item rather than provide an overall rating for the domain considering all items together as in the standard administration.

NPI-I Distress ${ }^{6}$. The observer rated the emotional distress (16) they experience in relation to the participant's behavior. Participants also completed the NPI-I Distress about their own behavior, referred to as Participant Distress.

Global outcome measures. Broad measures of general emotional and behavioral function were also collected from the three rater perspectives. Global Impression of Change (GIC) was completed by observers and participants. The observers and participants openly discussed their status and progress throughout the trial with each other and their study physician; however, completion of the GIC and the other measures was performed independently. The physician rated the Global Improvement subscale of the Clinical Global Impressions (CGI). ${ }^{7}$ The Global Improvement subscale is rated 1 through 7 ( 1 very much improvement and 7 very much worse). In scoring the CGI, the physicians performed a structured interview to solicit relevant information about general emotional and behavioral function (e.g., irritability, aggression, depression, impulsivity, awareness, initiation, and social interaction) from both the Observers and the Participants, and record their own direct observation and impression. The physicians then considered information obtained from the structured interview in assigning the GCI score.

Other measures included. Baseline Glasgow Outcome Scale-Extended (GOS-E), ${ }^{8}$ and baseline Beck Depression Inventory-II. ${ }^{9}$

\section{Statistical methods}

Baseline characteristics were compared between the treatment groups using the Student $t$ test to compare the means of variables measured on an interval scale (e.g., age) when appropriate to assume approximate normality and Wilcoxon rank sum test when not. Chi-square tests or Fisher exact tests were used for variables measured on a nominal scale (e.g., sex, race). The SAS ${ }^{\circledR}$ Enterprise Guide $^{\circledR} 5.1$ was used for analyses with a two-tailed $p$ value of less than 0.05 considered statistically significant. The $p$ value was adjusted for multiple comparisons within the domain of respondent, follow-up interval, and pre hoc versus post hoc analyses using the Holm sequential Bonferroni method. ${ }^{10}$

An intention-to-treat principle was used. The primary planned analysis compared the change in NPI-I Most Problematic (frequency $\times$ severity) score from baseline to day 28 and baseline to day 60 between the two groups using a Wilcoxon rank sum (MannWhitney) test for ordinal data. The same type of statistical analysis was performed on NPI-I Most Aberrant and NPI-Distress. The analyses were repeated using Per Protocol Principle to compare the amantadine and placebo groups with exclusion of those participants with $<80 \%$ pill count or failure to undergo follow-up NPI-I assessment.

The amantadine and placebo groups were compared on baseline characteristics including: age at enrollment, age at injury, years post-injury, number of TBIs, sex, race, cause of injury, GCS score, length of loss of unconsciousness, duration of post-traumatic amnesia, baseline Beck Depression Inventory-II, baseline GOS-E, baseline CGI Severity of Illness, baseline NPI-I Most Problematic score, baseline NPI-I Distress score, and study site. This was done using $t$ tests for interval data and nonparametric tests for ordinal data.

A pre-planned secondary analysis compared the percentages of participants considered to have meaningful change in irritability (a decrease of $\geq 3$ points) on NPI Most Problematic, and a post hoc analysis of NPI Most Aberrant from baseline to 28 days and baseline to 60 days between the two groups using the chi square. This meaningful change definition was used in the previous singlesite study and chosen based on consumer input and investigator clinical experience. Groups were additionally compared for differences in change in Observer and Participant Global Impression of Change and Physician-rated Clinical Global Impressions.

Trial replication will likely yield a smaller effect, even if the circumstances of the two trials were identical. ${ }^{11}$ The single-site study indicated that the probability of the treatment group showing a superior outcome to the control group was $0.70(p)=$ probability $(Y>X)=0.70)$. To adjust for this replication phenomenon in our power calculation, we assumed a slightly smaller effect size of $p "=0.63$ and a power of $80 \%$ (alpha $=0.05)$. Using these values, 78 participants would be needed in each group. Estimating an $8 \%$ loss to follow-up, the enrollment target was 84 in each of the two arms (total sample of 168).

\section{Results}

\section{Participants}

Of 324 persons screened, 168 were enrolled and randomized (86 in placebo and 82 in amantadine group; see Fig. 1). All but 11 (6\%; 4 in placebo and 7 in the amantadine group) completed the study. One participant required unmasking by the site principal investigator for medical management. The amantadine and placebo groups were well matched with respect to baseline factors (Table 1) with the exception of a small but statistically significant difference $(p=0.0492)$ in mean baseline GOS-E. Compliance (defined $a$ priori as $>80 \%$ pills taken as prescribed) was $88.5 \%$ in the amantadine group and $86.9 \%$ in the placebo group.

Although the presence of irritability was required for enrollment, observer distress was not required. All baseline Observer Distress scores were $>0$ with five ratings of 1 or 2 . For Participant Distress, 9 participants had scores of 0 and 21 had scores of 1 or 2 .

\section{Amantadine effect on irritability}

Observer ratings. Comparison of the change in day 28 and 60 NPI-I Most Problematic, Most Aberrant, and Distress ratings by observer are summarized in Table 2. There were large improvements in both groups; however, no statistically significant differences were noted between the groups for any of the observer NPI-I ratings at either follow-up interval. At day 60, 74.7\% in the amantadine group improved at least 3 points on the Observer NPI Most Problematic, compared with $68.3 \%$ in the placebo group $(p=0.3777)$. The other Observer NPI and Observer Global Impression of Change ratings similarly revealed large improvements without statistical significant between group differences. Per protocol analysis of Observer NPI-I Most Problematic including only those with $>80 \%$ pill compliance also revealed no significant difference between groups.

Participant ratings. Table 3 depicts comparisons of Participant NPI-I ratings. Participant ratings at day 28 were not statistically significant. For day 60, NPI-I Most Problematic $(p=0.0353)$ and Distress ratings ( $p=0.0362$ ), but not Most Aberrant, showed statistically significant group differences at day 60. After adjustment for multiple comparisons, however, the $p$ values were no longer significant. For illustrative purposes, the mean NPI-I Most Problematic scores at each assessment period for participants and observers are depicted in Figure 2. Large proportions in both groups improved at least 3 points without significant differences between 

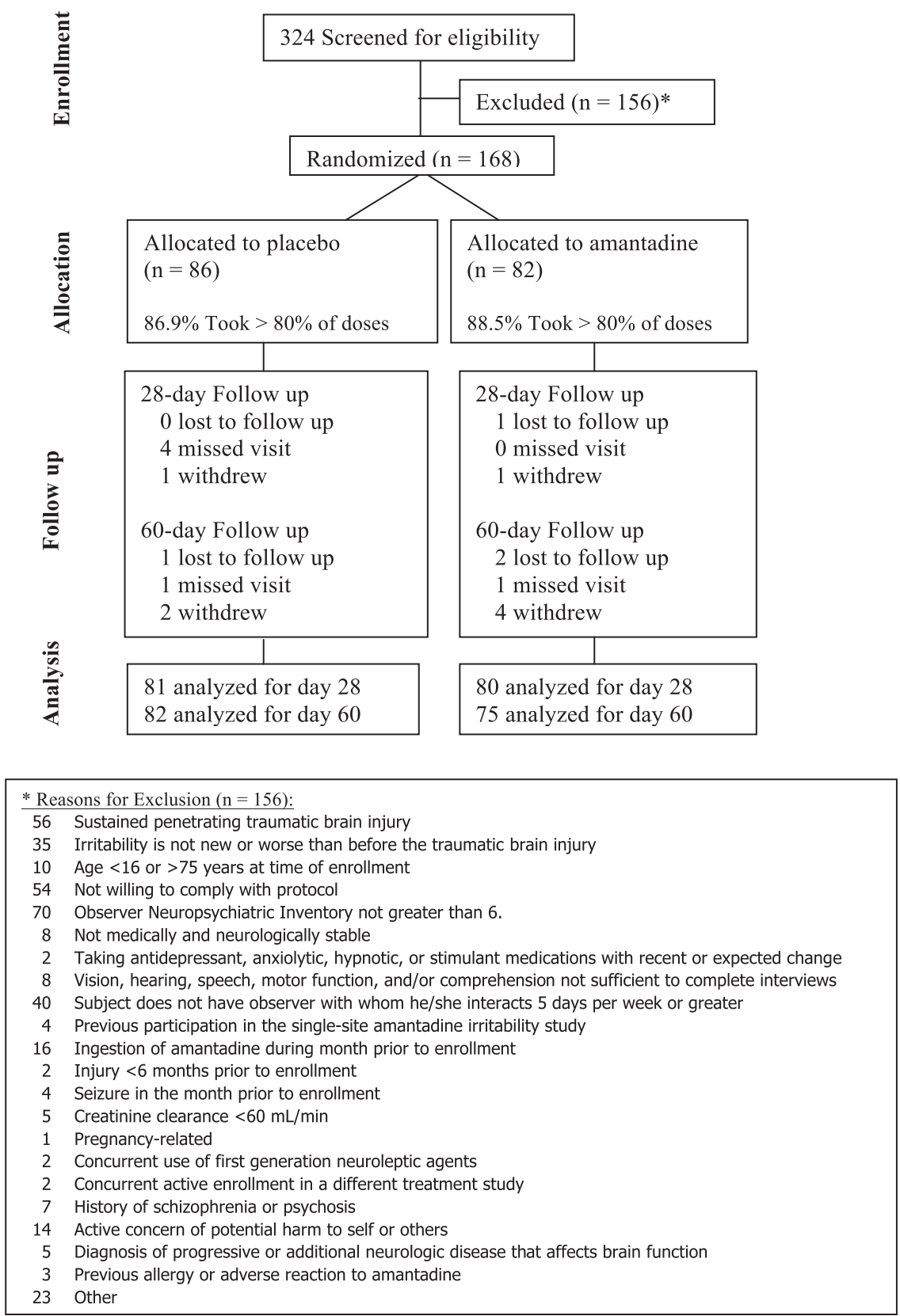

FIG. 1. Consort diagram showing study flow.

groups on participant rating. Group difference in Participant Global Impression of Change was not statistically significant.

Clinician ratings. Clinical ratings are summarized in Table 4, revealing greater Global Improvement for the amantadine group than the placebo group at Day $60(p=0.0354)$.

\section{Adverse events}

Amantadine was well tolerated among study participants. There were no significant differences between the two groups on withdrawals/lost or adverse events using the Fisher exact test. Adverse events are summarized in Supplementary Digital Content (supplementary data are available online at www.liebertpub.com/end).

\section{Discussion}

To our knowledge, this is the largest pharmacotherapy study for TBI irritability. While both groups showed robust improvement from the observer perspective, no statistically significant group differences were observed. The same was true for Participant ratings following adjustment for multiple comparisons. From the perspective of clinician, pre-specified analyses suggest a possible benefit of amantadine at day 60 , but not at day 28 . In summary, the null hypothesis cannot be rejected.

Increasingly experts ${ }^{12}$ in experimental design and analysis have advocated for reporting point and interval estimates for clinical trials in the context of cumulative experience in similar trials. Along these lines, a meta-analysis of the day 28 current findings 
Table 1. Baseline Participant Characteristics by Treatment Group

\begin{tabular}{|c|c|c|c|c|}
\hline Variable & Category & $\begin{array}{l}\text { Placebo }(\mathrm{n}=86) \\
\text { Number }(\%)\end{array}$ & $\begin{array}{l}\text { Amantadine }(\mathrm{n}=82) \\
\text { Number }(\%)\end{array}$ & $\mathrm{p}$ value \\
\hline Sex & Male & $74.4 \%$ & $80.5 \%$ & 0.3473 \\
\hline \multirow[t]{3}{*}{ Race } & Caucasian & $87.2 \%$ & $89.0 \%$ & 0.9359 \\
\hline & Black & $5.8 \%$ & $6.1 \%$ & \\
\hline & Other & $7.0 \%$ & $4.9 \%$ & \\
\hline Hispanic & Yes & $9.3 \%$ & $3.7 \%$ & 0.1394 \\
\hline \multirow{5}{*}{ Education } & Less than HS & $16.3 \%$ & $6.1 \%$ & 0.3472 \\
\hline & HS diploma & $30.2 \%$ & $37.8 \%$ & \\
\hline & Some college & $51.7 \%$ & $48.3 \%$ & \\
\hline & Bachelors or toward masters & $45.5 \%$ & $54.6 \%$ & \\
\hline & Masters and above & $7.0 \%$ & $7.3 \%$ & \\
\hline \multirow[t]{6}{*}{ Cause of injury } & Vehicular & $61.6 \%$ & $69.5 \%$ & 0.5513 \\
\hline & Fall & $18.6 \%$ & $13.4 \%$ & \\
\hline & Assault & $10.5 \%$ & $6.1 \%$ & \\
\hline & Sport-related & $3.5 \%$ & $1.2 \%$ & \\
\hline & Pedestrian & $3.5 \%$ & $7.3 \%$ & \\
\hline & Other & $2.3 \%$ & $2.4 \%$ & \\
\hline \multirow[t]{8}{*}{ Loss of consciousness duration } & $<1 \mathrm{~h}$ & $27.9 \%$ & $23.1 \%$ & 0.6845 \\
\hline & $\geq 1$ h but $<24 h$ & $15.1 \%$ & $12.8 \%$ & \\
\hline & $1-6 \mathrm{~d}$ & $16.3 \%$ & $24.4 \%$ & \\
\hline & $7-13 d$ & $8.1 \%$ & $6.4 \%$ & \\
\hline & $14-20 d$ & $9.3 \%$ & $12.8 \%$ & \\
\hline & $21-29 d$ & $10.5 \%$ & $9.0 \%$ & \\
\hline & $30-59 d$ & $9.3 \%$ & $6.4 \%$ & \\
\hline & $\geq 60 \mathrm{~d}$ & $3.5 \%$ & $5.1 \%$ & \\
\hline \multirow[t]{7}{*}{ Post-traumatic amnesia duration } & $<24 \mathrm{~h}$ & $9.6 \%$ & $11.5 \%$ & 0.9685 \\
\hline & $1-6 d$ & $22.9 \%$ & $11.5 \%$ & \\
\hline & $7-13 d$ & $4.8 \%$ & $10.3 \%$ & \\
\hline & $14-20 \mathrm{~d}$ & $9.6 \%$ & $14.1 \%$ & \\
\hline & $21-29 d$ & $12.1 \%$ & $10.3 \%$ & \\
\hline & $30-59 d$ & $15.7 \%$ & $23.1 \%$ & \\
\hline & $\geq 60 \mathrm{~d}$ & $25.3 \%$ & $19.2 \%$ & \\
\hline History $>1$ TBI & Yes & $18.6 \%$ & $13.4 \%$ & 0.3599 \\
\hline \multirow{5}{*}{ Total Glasgow Coma Scale score } & $3-8$ & $30.8 \%$ & $22.5 \%$ & 0.4672 \\
\hline & $9-12$ & $1.3 \%$ & $4.2 \%$ & \\
\hline & $13-15$ & $25.6 \%$ & $23.9 \%$ & \\
\hline & $\begin{array}{l}\text { Chemically paralyzed, chemically } \\
\text { induced coma, or intubated }\end{array}$ & $42.3 \%$ & $49.3 \%$ & \\
\hline & & Mean $(S D)$ & Mean $(S D)$ & \\
\hline \multicolumn{2}{|l|}{ Age at enrollment } & $38.23(12.36)$ & $40.18(12.67)$ & 0.2814 \\
\hline \multicolumn{2}{|c|}{ Age at injury } & $31.88(12.07)$ & $33.85(12.87)$ & 0.3803 \\
\hline \multicolumn{2}{|c|}{ Observer NPI irritability Most Problematic } & $8.35(2.13)$ & $8.56(2.36)$ & 0.7859 \\
\hline \multicolumn{2}{|c|}{ Participant NPI irritability Most Problematic } & $6.17(3.54)$ & $6.15(3.18)$ & 0.8593 \\
\hline \multicolumn{2}{|c|}{ Observer NPI irritability Most Problematic Distress } & $3.77(0.95)$ & $3.65(1.06)$ & 0.5421 \\
\hline \multicolumn{2}{|c|}{ Participant NPI irritability Most Problematic Distress } & $3.00(1.41)$ & $3.05(1.28)$ & 0.9427 \\
\hline \multicolumn{2}{|c|}{ Beck Depression Inventory-II Total } & $18.36(11.09)$ & $17.39(9.76)$ & 0.6705 \\
\hline \multicolumn{2}{|l|}{ Glasgow Coma Scale-Extended } & $5.05(1.38)$ & $5.51(1.19)$ & $0.0492 *$ \\
\hline
\end{tabular}

HS, high school, TBI, traumatic brain injury; SD, standard deviation; NPI, Neuropsychiatric Inventory.

*Statistically significant.

combined with the single-site study by Hammond and associates ${ }^{4}$ was performed (results depicted in Fig. 3) revealing an effect favoring amantadine over placebo. The implication of this metaanalysis would have been even more positive had we used day 60 results for the current trial in which those with more than a 2 point improvement on the Observer NPI-I Most Problematic were more frequent in the amantadine group by $+6.4 \%$ (95\% confidence interval: $-7.2 \%$ to $+20 \%$ ).

The positive day 60 effect among the participants (before adjustment for multiple comparisons) and clinicians is of interest when compared with the lack of significant differences at day 28 . Figure 2 shows that both groups had a substantial decrease in irritability by day 28 , with the amantadine group experiencing further improvement by day 60 . The NPI assessment of behavior over the previous month may not have allowed enough time for some to recognize the full benefit of behavior changes by day 28 . People often require several social interactions to acquire self-insights into behavioral change; clinicians are often impacted by participant perceptions in deriving their rating. In addition, the postulated impact of amantadine on dopamine, N-methyl-D-aspartate receptor 

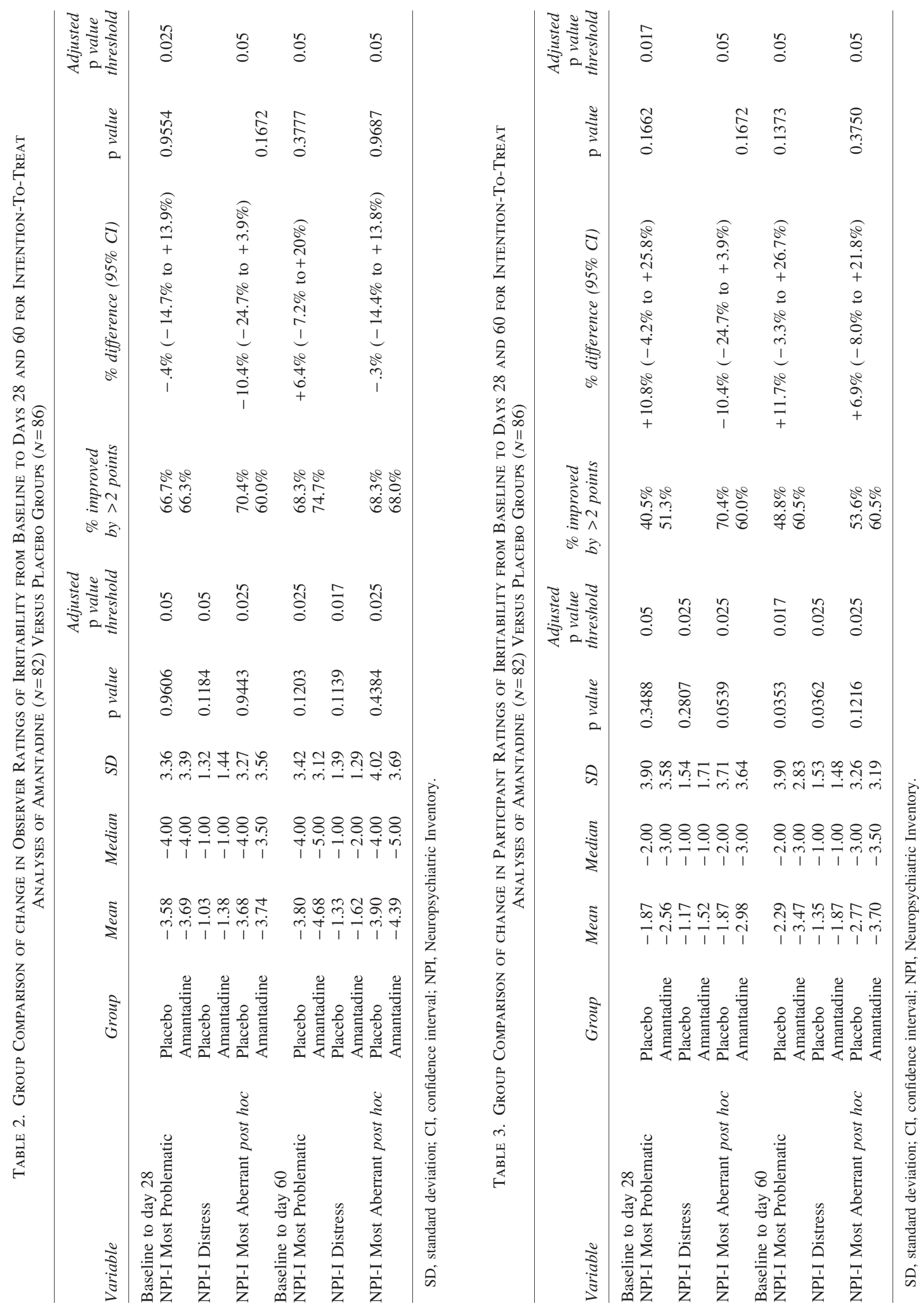


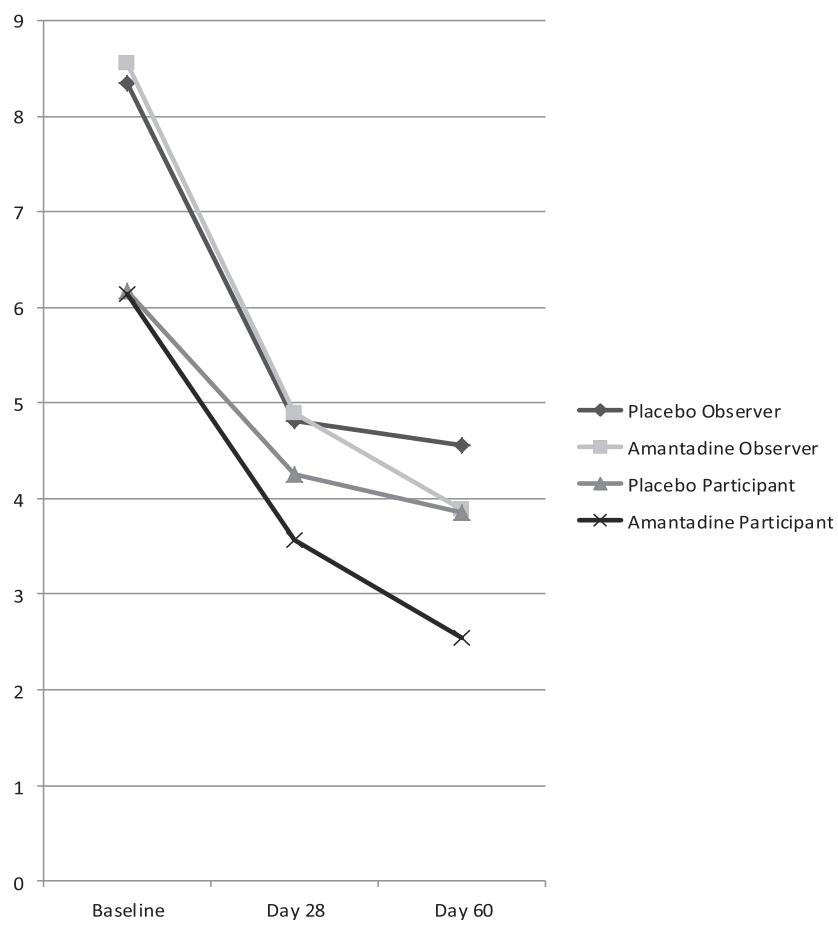

FIG. 2. Mean Observer and Participant ratings for Neuropsychiatric Inventory-Irritability Most Problematic at baseline, day 28, and day 60 .

regulation, and neurotrophic factors ${ }^{4,13}$ may have taken a longer time to fully impact the executive and inhibitory networks associated with reduced irritability.

Most notably, we found a large proportion of persons in both groups experienced significant symptom improvement (i.e., greater than 2 point NPI-I reduction in approximately $70 \%$ of both groups). This may be because of multiple factors. Resolution of irritability because of early recovery does not likely explain the changes in this cohort that was over 6 months post-injury. Only persons with Observer NPI-I Most Problematic score $>6$ at baseline were enrolled. While this admission criterion seems reasonable to assure only persons with the target symptom severity are studied, the large decline in both groups might have resulted from regression to the mean.

In the psychotherapy literature ${ }^{14}$ and depression drug studies, ${ }^{15}$ the impact of nonspecific effects, or factors common across therapies, such as therapeutic alliance and positive expectations, tend

\section{Table 4. Group Comparisons of Clinical Global IMPRESSIONS FOR GLOBAL IMPROVEMENT FROM BASELINE TO DAY 28 AND DAY 60} of Amantadine $(N=82)$ Versus Placebo Groups $(N=86)$

\begin{tabular}{llcccc}
\hline Variable & Group & Mean & Median & SD & p value \\
\hline $\begin{array}{l}\text { Global } \\
\quad \text { improvement }\end{array}$ & Placebo & 3.17 & 3.00 & 0.92 & 0.2410 \\
$\quad$ day 28 & & 2.94 & 3.00 & 1.14 & \\
$\begin{array}{l}\text { Global } \\
\quad \begin{array}{l}\text { improvement } \\
\text { day 60 }\end{array}\end{array}$ & Placebo & 3.01 & 3.00 & 1.08 & $0.0354^{*}$ \\
\hline
\end{tabular}

$\mathrm{SD}$, standard deviation.

*Statistically significant.

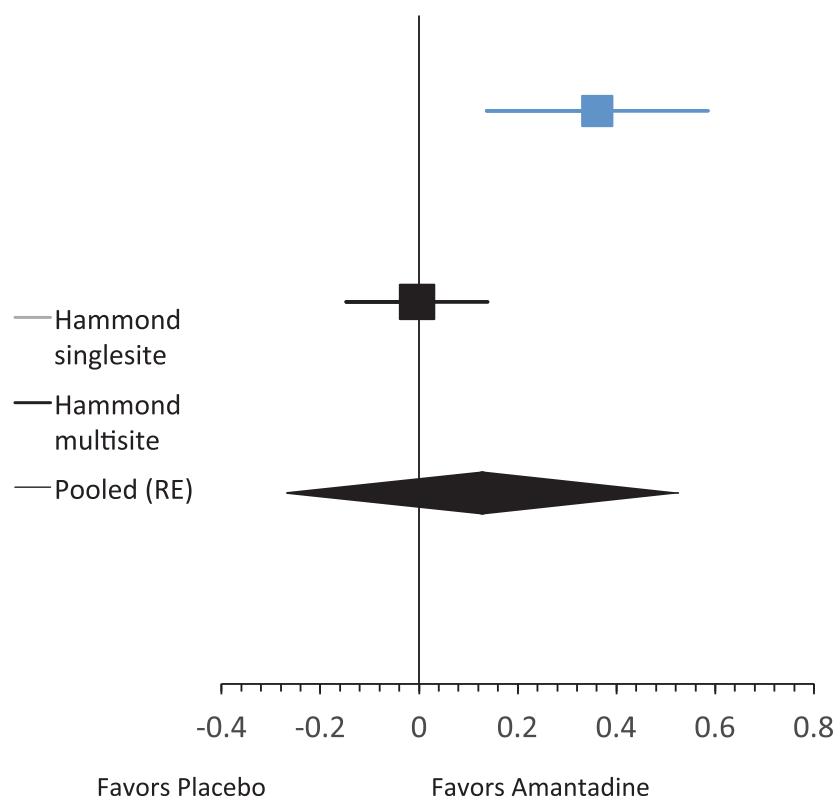

FIG. 3. Meta-analysis of difference in probability between treatment and control groups (risk difference) of $>2$ point decrease in Neuropsychiatric Inventory-I Observer Most Problematic at day 28.

to be much larger than specific effects of the intervention. ${ }^{16}$ Therapeutic alliance has become increasingly considered a potent contributing factor to positive outcomes in rehabilitation intervention studies. ${ }^{17,18}$ The investigators and study coordinators did not interact with participants and observers in an explicitly psychotherapeutic manner but did treat participants and observers with kindness and respect, offering support and encouragement to maintain their participation in the trial. The alliance between study personnel and participants may also have contributed to the large proportion of positive outcomes in both groups. The remarkably high compliance rate with study procedures and follow-up rates in the current study support such an explanation.

Simply monitoring a behavior consistently with participant awareness may result in a positive change in that behavior. ${ }^{19,20}$ Such a monitoring effect may have contributed to positive outcomes in both groups. Other nonspecific effects and expectations also likely played a role in the improvement apparent in all participants. Most persons with TBI and their caregivers receive no ongoing follow-up after inpatient medical stabilization and rehabilitation. $^{21}$ This lack of chronic care management and support may make this population particularly desperate for help and responsive to nonspecific effects. While we did not anticipate, monitor, or measure any of these factors, we speculate on their possible role in the large improvement seen in the control group to encourage consideration of such issues in future research.

The overriding message of this study appears to be that persons with TBI who demonstrate significant irritability will benefit from professional monitoring and attention to their problem, although recommendations for specific treatment remain elusive. The impact of nonspecific effects should not be underestimated, given that similar effects have been found to have neurobiological underpinnings. $^{22}$

Large nonspecific effects have been found in other TBI intervention studies and have been well noted in psychiatric research $^{23,24}$ These findings challenge the field to develop research 
methodologies to identify the incremental effects of active treatment layered on very large nonspecific effects. Development and use of interval-equivalent metrics that maximize sensitivity and specificity, assessing and covarying measurable nonspecific factors, such as therapeutic alliance, may provide another avenue for reducing the variance unaccounted for on the outcome variable.

To address large potential placebo effects, study designs that winnow out placebo responders and address impact on sample size should be considered and may be important future considerations. ${ }^{25}$ Such designs include Sequential Parallel Comparison Design and placebo run-in. Other considerations may include minimization of nonspecific effect, lengthened study duration, reduced number of study sites, and simplified study assessments.

The definition of irritability as a score $\geq 6$ on the observer rated NPI-I Most Problematic may be one of the sources of difficulty in establishing the impact of amantadine. This score is obtained on one occasion relating to behavior over a period before the scaling (retrospective) in an effort to establish a pattern of irritability that impacts function. Perhaps prospective measurement during the course of the study would provide a better baseline, and serial measure administration over time would provide a more reliable assessment of irritability. Although persons with NPI observer ratings of $<6$ were excluded, the correlation of NPI score with clinically significant irritability has not been firmly established. Consequently, it is possible that this measure and the manner administered allowed persons with mild irritability to be included.

Amantadine at $100 \mathrm{mg}$ twice daily is a clinically reasonable dose that appears to be well tolerated, although it may not be the optimal dose. This dose was chosen based on the first author's clinical experience, the previous single-site study that only tested this single dose, ${ }^{4}$ and a previous report of greater behavioral toxicity (including irritability) as the amantadine dose increases. ${ }^{26}$ Thus, moderate dosing was used for this study; however, dosing of amantadine for irritability still needs to be established. Subsequent investigation might assess higher dosing levels or use dose titration based on inadequate response.

Very large, simplified mega-trials of heterogeneous samples, which use randomization to equalize bias between study arms, is another approach for studying a problem such as treatment of postTBI irritability with low-risk treatments such as amantadine. Simplified mega-trials are massive randomized clinical trials that enroll thousands of subjects to allow the statistical power to assess the advantages of moderately and marginally effective but worthwhile treatments. Such studies use broad eligibility criteria, highly simplified protocol, and clearly defined and meaningful endpoints. ${ }^{27}$ Mega-trials, however, require sufficient preparatory investigation of the therapeutic intervention to allow appropriate protocol simplification while minimizing impact on experimental control, and, as discussed above, some of this preliminary work, such as determining optimal dosing, has yet to be completed for the use of amantandine to treat persons with post-TBI irritability.

While this approach may have advantages, it would also be expensive and require many sites, which runs a high risk to data and protocol integrity, including the use of nontrial interventions. In addition, the treatment of persons with irritability is often a series of ongoing treatments further complicating the application of the simplified mega-trial. Rather than increasingly larger trials, an "individualized medicine" approach in which subjects studied are also genetically profiled to allow determination of differences between responders and nonresponders holds promise for future study of pharmacologic interventions in this and other applications.

\section{Limitations}

Subjective measures were used because irritability generally expresses itself sporadically in the home setting, precluding use of more objective measures. Observers represented a variety of different roles and relationships to the person with TBI. Observers had close enough proximity to witness the participant's behaviors, but were not required to have a caregiving role or bear the brunt of the irritable behaviors. In addition, there was no required level of Observer Distress over the behaviors. Some observers did not have marked distress at baseline, again raising the possibility that an NPI score $>6$ does not represent significant irritability in all cases. On the other hand, some observers appeared more tolerant of irritability, and the change in GIC suggests that from an objective evaluator's perspective, there was a clinically significant change in irritability for the treatment group. In addition, not all observers were caregivers per se because persons with chronic brain injury, particularly ones meeting the inclusion criteria for this study, often have emotional, behavioral, and cognitive impairment, yet are also often quite independent. Such factors may have contributed to the lack of significant differences on NPI-I Distress.

\section{Conclusion}

While observers in both groups reported large improvements, significant group differences were not found for the primary outcome (observer ratings) at either day 28 or 60 . From the perspective of persons with TBI (before multiple comparison adjustment but not after) and their clinicians (based on a singular global outcome metric), pre-planned secondary analyses revealed amantadine $100 \mathrm{mg}$ every morning and noon may reduce irritability and the perceived distress associated with irritability at 60 days posttreatment. The findings of improvement in both groups, as well as the significant improvement from clinician and participant perspectives may warrant further investigation.

\section{Acknowledgment}

The research reported in this article was supported by US Department of Education, Office of Special Education and Rehabilitative Services, National Institute on Disability and Rehabilitation Research grant H133A080035. This support included the funds to purchase amantadine. The study sponsor had no role in the design and conduct of this study; the collection, management, analysis, and interpretation of the data; or the preparation review, or approval of the manuscript.

The authors gratefully acknowledge the work of the Data Safety and Monitoring Board, study site co-investigators (Lori Grafton, MD, Shilpa Kasuganti, MD, Mel Glenn, MD, Jennifer Zumsteg, MD, Christian Shenouda, MD, Joseph Rosenthal, MD), as well as study coordinators and project staff (Peggy Cook, Tami Guerrier, CRTS, Elena Gillespie, PhD, Jay Bogaards, MA, Laura A. Burns, MBA, Judith A. Frazier, RN, MEd, Iris M. Monge, Leslie Kempthorne, BA, BS, Monica A. Lichi, MS Ed, PC, MBA, Colleen M. Sheehan, MS, Laura VanArsdale, MS).

Trial Registration: clinicaltrials.gov Identifier: NCT00779324; http://www.clinicaltrials.gov/ct2/show/NCT00779324?term = irritability\&rank $=6$

\section{Author Disclosure Statement}

The participant institutions received funding for the project from the US Department of Education, Office of Special Education and Rehabilitative Services, National Institute on Disability and 
Rehabilitation Research grant H133A080035. In addition, Dr. Hammond received personal fees from Avanir, nonfinancial support from Eli Lilly, personal fees from Mayo Clinic, personal fees from Baylor College of Medicine, personal fees from Rehabilitation Institute of Chicago, personal fees from Galveston Brain Injury Conference, and stock ownership (Stock ownership in health care companies include: ABBVIE INC SHS [\$34,247 market value], ELI LILLY \& CO $[\$ 14,535]$, GLAXOSMITHKLINE PLC ADR [\$23,794], Exchange Traded Funds [84,545], and Mutual Funds $[\$ 47,095])$, outside the submitted work. For the remaining authors, no competing financial interests exist.

\section{References}

1. Hammond, F.M., Davis, C.S., Whiteside, Y.O., Philbrick, P., and Hirsch, M.A. (2011). Marital adjustment and stability following traumatic brain injury: a pilot qualitative analysis of spouse perspectives. J. Head Trauma Rehabil. 26, 69-78.

2. Kilmer R.P., Demakis G.J., Hammond F.M., Grattan, K.E., Cook, J.R., and Kornev, A.A. (2006). Use of the Neuropsychiatric Inventory in traumatic brain injury: A pilot investigation. Rehabil Psychol 51, 232 238.

3. Ciurli, P., Formisano R., Bivona, U., Cantagallo, A., and Angelelli, P. (2011) Neuropsychiatric disorders in persons with severe traumatic brain injury: prevalence, phenomenolgy, and relationship with demographic, clinical, and functional features. J. Head Trauma Rehabil. 26, 116-126.

4. Hammond, F.M., Bickett, A.K., Norton, J.H., and Pershad, R. (2013). Amantadine hydrochloride in the reduction of chronic traumatic brain injury irritability and aggression. J Head Trauma Rehabil 29, 391-399.

5. Cummings, J.L., Mega, M., Gray, K., Rosenberg-Thompson, S., Carusi, D.A., and Gornbein, J. (1994). The Neuropsychiatric Inventory: comprehensive assessment of psychopathology in dementia. Neurology 44, 2308-2314.

6. Kaufer, D.I., Cummings, J.L., Christine, D., Bray, T., Castellon, S., Masterman, D., MacMillan, A., Ketchel, P., and DeKosky, S.T. (1998). Assessing the impact of neuropsychiatric symptoms in Alzheimer's disease: the Neuropsychiatric Inventory Caregiver Distress Scale. J. Am. Geriatr. Soc. 46, 210-215.

7. Health Services/Technology Assessment Texts: The Clinical Global Impressions Scale. http://www.ncbi.nlm.nih.gov/books/bv.fcgi?rid= hstat

8. Wilson, J.T., Pettigrew, L.E., and Teasdale, G.M. (1998). Structured interviews for the Glasgow Outcome Scale and the Extended Glasgow Outcome Scale: guidelines for their use. J. Neurotrauma 15, 573-585.

9. Beck, A.T., Steer, R.A., and Brown, G.K. (1996). Manual for the Beck Depression Inventory-II. Psychological Corporation: San Antonio, TX.

10. Holm, S. (1979). A simple sequentially rejective multiple test procedure. Scand J Stat 6, 65-70.

11. Berry, D.A. (1996). When is a confirmatory randomized clinical trial needed? J. Natl. Cancer Inst. 88, 1606-1607.

12. Cumming, G. (2012). Understanding the New Statistics: Effect Sizes, Confidence Intervals, and Meta-Analysis. Routledge: New York.

13. Peeters, M., Maloteaux, J.M., and Hermans, E. (2003). Distinct effects of amantadine and memantine on dopaminergic transmission in the rat striatum. Neurosci. Lett. 343, 205-209.
14. Wampold, B.E. (2001). Great Psychotherapy Debate: Models, Methods, and Findings. Lawrence Erlbaum Associates: Mahwah, N.J.

15. Rief, W., Nestoriuc, Y., Weiss, S., Welzel, E., Barsky, A.J., and Hofmann, S.G. (2009). Meta-analysis of the placebo-response in antidepressant trials. J. Affect. Disord. 118, 1-8.

16. Miciak, M., Gross, D.P., and Joyce A. (2012). A review of the psychotherapeutic 'common factors' model and its application in physical therapy: the need to consider general effects in physical therapy practice. Scand. J. Caring Sci. 26, 394-403.

17. Evans, C.C., Sherer, M., Nakase-Richardson, R., Mani, T., and Irby, J.W., Jr. (2008). Evaluation of an interdisciplinary team intervention to improve therapeutic alliance in post-acute brain injury rehabilitation. J. Head Trauma Rehabil. 23, 329-338.

18. Schönberger, M., Humle, F., and Teasdale, T.W. (2006). The development of the therapeutic working alliance, patients' awareness and their compliance during the process of brain injury rehabilitation. Brain Inj. 20, 445-454.

19. Bird, E.L., Baker, G., Mutrie, N., Ogilvie, D., Sahlqvist, S., and Powell, J. (2013) Behavior change techniques used to promote walking and cycling: a systematic review. Health Psychol 32, 829-838.

20. Spahn, J.M., Reeves, R.S., Keim, K.S., Laquatra, I., Kellogg, M., Jortberg, B., and Clark, N.A. (2010). State of the evidence regarding behavior change theories and strategies in nutrition counseling to facilitate health and food behavior change. J. Am. Diet. Assoc. 110, 879-891.

21. Masel, B.E., and DeWitt, D.S. (2010). Traumatic brain injury: a disease process, not an event. J Neurotrauma 27, 1529-1540.

22. Eknoyan, D., Hurley, R.A., and Taber, K.H. (2013). The neurobiology of placebo and nocebo: how expectations influence treatment outcomes. J. Neuropsychiatry Clin. Neurosci. 25, vi-254.

23. Silver, J.M., Koumaras, B., Chen, M., Mirsky, D., Potkin, S.G., Reyes, P., Warden, D., Harvey, P.D., Arciniegas, D., Katz, D.I., and Gunay I. (2006). Effects of rivastigmine on cognitive function in patients with traumatic brain injury. Neurology 67, 748-755.

24. Ehde, D., Fann J., Bell K., Dikmen, S, Bombardier, C., and Temkin, N. (2014). Clinical trials of behavioral interventions in neurologic patients developing evidence. Presented at the 42nd Annual Symposium of the International Neuropsychological Society. February 2014, Seattle, Washington.

25. Fava, M., Evins, A.E., Dorer, D.J., and Schoenfeld, D.A. (2003). The problem of the placebo response in clinical trials for psychiatric disorders: culprits, possible remedies, and a novel study design approach. Psychother Psychosom 72, 115-127.

26. Gualtieri, C.T., and Evans, R.W. (1988). Stimulant treatment for the neurobehavioral sequelae of traumatic brain injury. Brain Inj. 2, 273290.

27. Peto R., Collins R., and Gray, R. (1995). Large-scale randomized evidence: large, simple trials and overview of trials. J. Clin. Epidemiol. 48, 23-40.

Address correspondence to:

Flora M. Hammond, MD

Indiana University School of Medicine Rehabilitation Hospital of Indiana Carolinas Rehabilitation 4141 Shore Drive Indianapolis, IN 46254

E-mail: Flora.hammond@rhin.com 